\title{
Accelerometer Placement for Posture Recognition and Fall Detection
}

\author{
Hristijan Gjoreski, Mitja Luštrek, Matjaž Gams \\ Department of Intelligent Systems \\ Jožef Stefan Institute \\ Jamova cesta 39, 1000 Ljubljana, Slovenia \\ E-mail: \{hristijan.gjoreski, mitja.lustrek, matjaz.gams\}@ijs.si
}

\begin{abstract}
This paper presents an approach to fall detection with accelerometers that exploits posture recognition to identify postures that may be the result of a fall. Posture recognition as a standalone task was also studied. Nine placements of up to four sensors were considered: on the waist, chest, thigh and ankle. The results are compared to the results of a system using ultrawideband location sensors on a scenario consisting of events difficult to recognize as falls or non-falls. Three accelerometers proved sufficient to correctly recognize all the events except one (a slow fall). The location-based system was comparable to two accelerometers, except that it was able to recognize the slow fall because it resulted in lying outside the bed, whose location was known to the system. One accelerometer was able to recognize only the most clear-cut fall. Two accelerometers achieved over 90\% accuracy of posture recognition, which was better than the location-based system. Chest and waist accelerometers proved best at both tasks, with the chest accelerometer having a slight advantage in posture recognition.
\end{abstract}

Keywords - Ambient intelligence, fall detection, posture recognition, activity recognition, accelerometers, accelerometer placement, classification.

\section{INTRODUCTION}

There are many studies describing new ways of improving the life of elderly. Improving the quality of life of Europe's increasing elderly population is one of the most pressing challenges facing our society. Nearly $14 \%$ of the EU population is over 65 and this figure is expected to double by 2050 [8]. By then Europe will have 80 million elderly citizens who should continue to play an active role in our society, despite limitations which the ageing process often brings. To cope with this situation, intelligent systems and techniques are being developed, which can give these people self-confidence to actively and independently live their life despite their age limitations.

The detection of falls is important task in ambient assisted living. Furthermore, activity/posture recognition is an essential component in such systems. In this paper we present our work on the detection of falls and alarming situations using wearable accelerometers. Fall detection is improved by body posture recognition. In posture recognition we were focused on seven basic body postures. We also investigated the performance of posture recognition and fall detection with different numbers of accelerometers ( 1 to 4 ) and different placements on the body (chest, waist, ankle and thigh). This way we showed the tradeoff between the intrusiveness of the system and the achieved accuracy. The final system should be as non-intrusive as possible (fewer wearable sensors), but still accurate enough to detect each fall. Eventually a comparison with a system based on location sensors was made. This system was developed in a European FP7 project - Confidence [11].

\section{RELATED WORK}

Lots of studies in ambient intelligence, particularly in ambient assisted living are focused on detecting alarming situations using different kinds of technical equipment. Many of the studies on human body posture analysis and fall detection use image processing, location sensors or accelerometers. However, each of these approaches has certain problems.

The image processing approach [6] has several operational complexities. One of them is the process of installation of the camera in each room we want the system to work. Another issue is limitation of functioning only in indoor environment. Also common disadvantages are the low image resolution and target occlusion. And probably the biggest issue in this approach is the user privacy. The user has to accept the fact that a camera will record him/her.

Some researchers have tried to analyze the body posture and fall detection using location sensors [3, 9, 10, 11]. The problem with this approach is the variation of the sensors precision (not constant rate of distance error) and price of the sensors. For reasonable results high precision is required (Ubisense 2011). High precision means high cost of sensors. This price is many times higher than the price of accelerometers. In addition, accelerometers are far more commercially available and nowadays they can be found almost in any smart phone. Similarly to the image processing approach, these location systems are limited to indoor environment and require installation in each room where we want the system to work. The advantage of location sensors compared to accelerometers is knowing the location of the person. That can be seen in our comparison with the locationbased system [11] presented in the fall detection results section. 
Another way of formulating posture recognition and fall detection task is by using accelerometers.

The most common approach for posture recognition is the data mining $[1,2]$. In a narrow sense it can be interpreted as a pattern recognition problem [1]. Therefore good attributes that explain the body posture are essential in this approach. Usually the results are presented in detection of the whole process of the activity/posture (e.g. lying for 20 seconds as one sample). In our work we tried to predict each data sample and the results are presented that way.

Also there are studies that use manually created algorithms to formulate the accelerometer based posture recognition task [13]. With this approach the achieved accuracies are very good (around 99\%), but it requires using multiple accelerometers (6).

Most studies on fall detection use accelerometers (which measure linear acceleration) and gyroscopes (which measure angular velocity). Some researchers used machine learning instead of threshold-based algorithms [14, 15]. In these 2 approaches they used a triaxial accelerometer worn on the waist. Using SVM machine learning algorithm on various features derived from accelerations, they detected falls with $96.7 \%$ and $100 \%$ accuracy, respectively.

Typical approach is detection of falls by applying thresholds to accelerations, velocities and angles [7]. In [12] they used a 3-axis accelerometer worn on the chest; by applying a simple threshold to the acceleration, they detected falls with 98.9\% accuracy. In [4] they used a 3-axis accelerometer worn on the waist; by applying thresholds to the acceleration, they detected a potential fall and the activity/posture after the fall, resulting in $100 \%$ accurate fall detection.

Of particular interest to us is the work in [5]. They used two 3-axis accelerometers and gyroscopes worn on the chest and thigh; by applying thresholds to accelerations, angular velocities and angles, they detected a potential fall and the activity/posture after the fall, resulting in $90.1 \%$ accurate fall detection. The lower accuracy compared to the previous work is most likely due to the more difficult test data: their method sometimes failed on lying down quickly and on two atypical fall types. Exactly such situations are tested in this paper. Fall detection using accelerometers may appear straightforward, but detecting all types of falls is challenging. Most of the researches show only accuracy in detecting fast falls. Because of that, having a good testing scenario which includes all kinds of falls is one of the key steps in fall detection procedure. In our approach we were interested in different alarming situations that were more difficult to detect only acceleration signal: falling slowly (e.g. losing consciousness) and sliding from chair (quickly and slowly). In addition, we tested sitting down quickly. All these events are included in the test scenario.

\section{PROBLEM DESCRIPTION}

In this section we describe the hardware components used in this research, and the architecture of our system for posture recognition and fall detection.

\section{A. Hardware}

The sensors used in this research were four 3-axis accelerometers. A 3-axis accelerometer is a sensor that returns a real-valued estimate of the acceleration along the $\mathrm{x}, \mathrm{y}$ and $\mathrm{z}$ axes from which velocity and orientation angle can also be estimated. Accelerometers measure the acceleration and output the projections of the acceleration vector represented in a three dimensional coordinate system. Each accelerometer has its own coordinate system and gives the relative vector projections. Because of the Earth's gravity, all objects experience a gravitational pull towards the Earth's center. The acceleration unit of the pull is referred to as $g$ or $g$ force. Consequently all objects are subject to $1 \mathrm{~g}$ acceleration. When the accelerometer is at rest, only Earth's gravity is measured. Accelerometers can be used as motion detectors as well as for body-posture recognition and fall detection. Acceleration sensors used in this research have data sampling frequency of $6 \mathrm{~Hz}$. This is not a high frequency, but it gives enough information for our final goal and makes the system more compact and portable even on devices with low memory and low processing power. It is also equal to the sampling frequency of the location sensors we used for comparison.

\section{B. System Architecture}

The posture recognition and fall detection process described in this paper is divided in several phases (Figure 1). It begins with the real world in which wearable accelerometers are affixed to a person's body. The next phase is the sensory part. Accelerometers sample the signal and send the raw data to the software attribute extraction module. The software analyzes the raw data and extracts new attributes. To solve the problem of posture recognition and detecting fall situations two software modules were developed: data mining body posture recognition and fall detection. Each of these modules gives an output: the real time posture of the body and a fall or non-fall event.

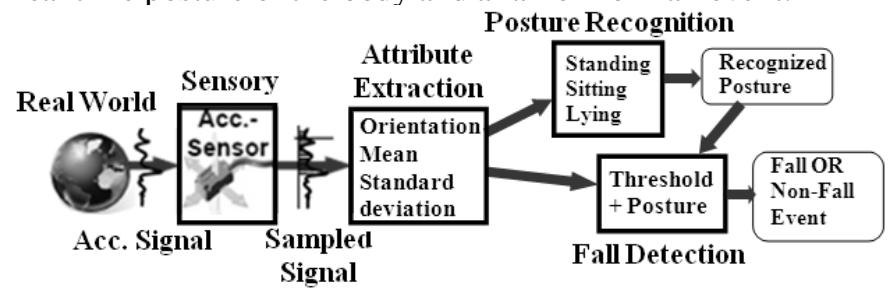

Figure 1: System Architecture

The data mining posture recognition module classifies the acceleration data samples into 7 predefined postures. Five of the postures were chosen to be common and general: standing, sitting, lying, standing up and going down (which includes sitting down, lying down or falling). Two of the postures are more specific and are related to fall detection: on all fours and sitting on the ground. The process of walking is included into posture standing, therefore standing can be a static or a dynamic posture.

As we already mentioned, each accelerometer experiences the gravitational acceleration. This is an important reference for calculating sensor orientation angle and distinguishing different body postures. The orientation angle is extracted as the angle between the acceleration vector ( $g-$ if the posture is static) and each of the axis unit vectors ( $\mathrm{x}, \mathrm{y}$ and $\mathrm{z}$ ). Using 
these 3 angles we have a unique sensor space orientation. With the extraction of the orientation angle attribute, the data mining algorithm can easily distinguish static postures that have different sensor orientation angles. For instance the chest accelerometer is perpendicular to the ground for standing and sitting postures, but parallel to the ground for lying and on all fours postures. Because of such similarities and differences in the orientation of the sensors the body postures can be recognized. Also a special movement attribute is extracted for distinguishing dynamic from static postures. This attribute captures the changes in the acceleration vector; the greater the changes, the fiercer the motion.

The fall detection module is focused on the detection of fall events. The first algorithm in this module detects high accelerations. It is a threshold-based algorithm which has some improvements for reducing false alarms. The second algorithm uses the information from posture-recognition module as input. It analyzes the recognized postures and decides if a fall occurred.

The number and placements of the accelerometers on the body can affect the recognition of particular postures. Placing an accelerometer on the thigh can help distinguishing sitting (accelerometer is parallel to the ground) and standing (accelerometer is perpendicular to the ground), but distinguishing sitting and lying is a problem. On the other hand, an accelerometer on the chest can distinguish sitting and lying, but has problems with standing and sitting. By combining these two accelerometers the algorithm was able to distinguish each of the discussed postures. Because of situations like this, we decided to compare the results using different numbers of accelerometers and different body placements. The idea is to use as few sensors as possible to maximize the user's comfort, but to use enough of them to achieve satisfactory performance. It is very important that the accelerometers are firmly fixed, because the orientation of the sensor is the most important feature in posture recognition. The body placements of the accelerometers were chosen to be: chest, waist, right thigh and the right ankle. The chest and waist positions are common in fall detection, so we wished to compare them.

\section{METHODS}

In this section we describe the methods and algorithms used in each of the modules in our system. Because we use data mining classification techniques for body posture recognition, the data collecting is one of the main steps. We use supervised classification techniques, therefore the data had to be labeled with the appropriate posture. As the raw data sample was received from the sensors, firstly it was labeled with the appropriate class value (posture) and afterwards saved in a database together with the correct posture. This was done manually while recording. We should note that because the labeling was done online, small delays in labels could happen. Therefore, successive activities (e.g. standing -> going down -> lying) may have some samples mislabeled at the beginning and at the end of each posture/activity. This can affect the recognition accuracy especially for short postures/activities (going down or standing up) that do not have lots of samples.
In addition, the border between two successive postures is to some degree subject to interpretation.

\section{A. Body Posture Recognition Module}

The real-time posture recognition module uses a classification model to classify (recognize) every data sample that is received from the accelerometers. Therefore good attributes that describe the person's posture are essential for successful classification. The first set of attributes are the raw acceleration vector projections (3 attributes for each accelerometer) $a_{\mathrm{x}}, a_{\mathrm{y}}$ and $a_{\mathrm{z}}$. Additional 18 attributes are extracted for each accelerometer:

- Length of the acceleration vector (1 attribute)

- Orientation angles for each axis - x, y and $\mathrm{z}$ (3 attributes)

- Statistical attributes for each axis and for the length of the acceleration vector

- Mean Value (4 attributes)

○ Root Mean Square (4 attributes)

- Standard Deviation (4 attributes)

- Movement detection attributes (2 attributes)

The first derived attribute is the length (module) of the acceleration vector. It is a simple but very useful attribute, which is also used further in the process of extraction of new attributes. Its definition is:

$$
\text { length }=\sqrt{a_{\mathrm{x}}^{2}+a_{\mathrm{y}}^{2}+a_{\mathrm{z}}^{2}} .
$$

During static postures this attribute is constant with the value equal to the Earth's gravity $(\mathrm{M}=1 \mathrm{~g})$. Otherwise in dynamic activities the acceleration vector is changing the direction and its module.

The most important characteristics for static body posture recognition are the orientation angles of the accelerometer. The orientation angles are calculated as the angles between the actual acceleration (Earth's gravity for static postures) and each of the axes ( $\mathrm{x}, \mathrm{y}$ and $\mathrm{z}$ ). For instance, the angle $\varphi_{x}$ between the acceleration vector and the $\mathrm{x}$ axis (perpendicular to the ground) is computed as follows:

$$
\cos \varphi_{x}=\frac{a_{x}}{\sqrt{a_{\mathrm{x}}^{2}+a_{\mathrm{y}}^{2}+a_{\mathrm{z}}^{2}}} .
$$

This attribute improves the classification for static postures that have different sensor angle orientations. Since each person has his/her characteristic posture and each accelerometer may not always be worn in exactly the same way, a method for the adaptation to the user is performed. At the beginning of each recording for each person there is initialization (normalization) period of 15 seconds. The average orientation angle of posture standing was measured as $\varphi_{0}$. The difference between the "ideal" standing orientation angle (e.g. $180^{\circ}$ for the $\mathrm{x}$ axis) and $\varphi_{0}$ was calculated as $\varphi_{\text {diff }}=$ $\varphi_{\text {ideal }}-\varphi_{0}$. After the normalization period, to each newly calculated orientation angle $\varphi_{i}$ the difference angle $\varphi_{\text {diff }}$ is added and finally the normalized angle is calculated as $\varphi_{\text {normal }}$ $=\varphi_{i}+\varphi_{\text {diff. }}$ This adaptation procedure is performed for each axis for each accelerometer. Without this adaptation technique the model and the results were person dependable. There was a big difference in the accuracy for people wearing the accelerometers in slightly different way. 
A sliding window is used for calculation of the statistical attributes. The current data sample and 5 past data samples are combined in one window. The window size is chosen to be 6 data samples ( 1 second interval) because in this task there are short-lasting activities that the system should detect (going down, standing up). The first attribute from this group is the Mean value of the data in the window. This attribute is actually performing a low-pass filter to the raw data. The filtered data is smoother and has fewer changes. This is a good feature for the posture recognition process. The mathematical definition for the $\mathrm{x}$ axis is:

$$
\operatorname{Mean}_{x}=\frac{\sum_{i=1}^{n} a_{x}^{i}}{n} .
$$

The number of data samples $n$ is 6 ( 1 second window size). The variable $a_{x}^{i}$ is the acceleration along $\mathrm{x}$ axis. Using the same formula, mean values for other axes $M_{e a n_{\mathrm{y}}}$ and $M e a n_{\mathrm{z}}$ are calculated. Also the mean value for the length of the acceleration vector $M e a n_{\text {length }}$ is calculated. A similar approach is used for the Standard deviation and the Root mean square attributes.

The Root Mean Square is a similar attribute to the Mean value, but it is useful when the observed value is varying above and below zero. That is the case in our acceleration values. Depending of the orientation of the accelerometer, the values can be positive or negative (e.g. $+g$ or $-g$ ). The RMS for the length of the acceleration vector is computed as follows:

$$
R M S_{\text {length }}=\sqrt{\frac{\sum_{i=1}^{n} \text { length }_{i}^{2}}{n}} .
$$

The variable length $h_{i}^{2}$ is the square of the length of the acceleration vector for the current member in the sum. Similarly the $R M S_{\mathrm{x}}, R M S_{\mathrm{y}}$ and $R M S_{\mathrm{z}}$ were calculated.

The Standard Deviation attribute is good for distinguishing long-lasting static postures/activities from transitional postures/activities. It can detect when the movement of the accelerometer is intense. The mathematical definition for the length of the acceleration vector is:

$$
S T D_{\text {length }}=\sqrt{\frac{\sum_{i=1}^{n}\left(\text { length }_{i}-\overline{\text { length }^{2}}\right.}{n}} .
$$

The variable lengthis the length for the current member in the sum and $\overline{l e n g t h}$ is the mean value in the current window. Also the standard deviation for each of the axes was calculated: $S T D_{\mathrm{x}}, S T D_{\mathrm{y}}$ and $S T D_{\mathrm{z}}$.

When a person's body is static, the accelerometers respond only to the gravity, producing a constant $1 \mathrm{~g}$ total acceleration. During motion the accelerometers produce changing acceleration signal and the fiercer the motion, the greater the change in the signal. Using these changes in the acceleration vector an attribute is computed for the detection of sensor movement - Acceleration Vector Changes (AVC). AVC value of this attribute increases as the accelerometer is in movement (walking, going down, standing up etc.). This attribute takes in consideration the last 2 seconds of data (12 data samples). It sums up the last 12 differences of lengths of the acceleration vector and divides the sum with the time interval (2 seconds) of the data. AVC is computed as follows:

$$
A V C=\frac{\sum_{i=0}^{n-1} \mid \text { length }_{i+1}-\text { length }}{i} .
$$

$T_{0}$ is the time stamp for the first data sample in the window and $T_{n}$ is the time stamp of the last data sample. With this attribute the movement of the person can be detected: it distinguishes static from dynamic postures. A boolean (true/false) attribute, which compares the AVC attribute value to a threshold, is also computed. If the value is above the threshold, the boolean attribute is true, otherwise it is false. The threshold value is 0.0015 and it was chosen empirically after series of tests on recordings different from the test ones.

All these 21 attributes were extracted for each accelerometer and collected together in one attribute vector. This vector was passed through to the classification model, which tried to predict the appropriate posture. The classification model was previously trained. Because we recorded 11 people, the model was trained on 10 people and tested on the remaining person. This procedure was repeated for each person. The decision which classification algorithm to be used was made after evaluating the results in the Weka toolkit. Several commonly used classification algorithms were analyzed: Naïve Bayes, SVM, J48 and Random Forest. The algorithm that achieved the best results for almost all postures was Random Forest.

\section{B. Fall Detection Module}

The second module is focused on the detection of fall events. In this module two acceleration-based algorithms are used. The first one detects high accelerations using only one accelerometer (chest or waist). It is a threshold-based algorithm and has some improvements for reducing false alarms. The acceleration pattern during a typical fall is a decrease in acceleration followed by a fast increase. This is shown in Figure 2. The reason for this pattern is that the acceleration at rest is $1 \mathrm{~g}$ and during free fall $0 \mathrm{~g}$. When a person starts falling, the acceleration decreases from $1 \mathrm{~g}$ to around $0.5 \mathrm{~g}$ (perfect free fall is never achieved). Upon the impact with the ground, a short increase in the acceleration is measured.

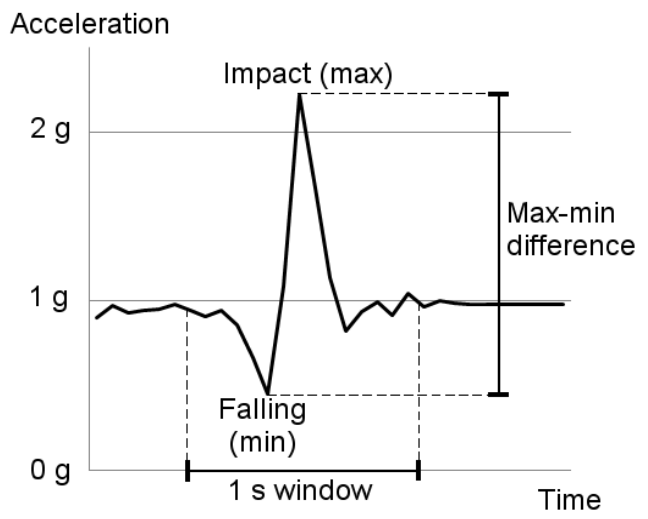

Figure 2: Acceleration pattern during a fall. 
To detect falls with a threshold, we used the length of the acceleration vector, which means that we ignored the orientation of the accelerometer. The first idea was to use a simple threshold that will detect only the high acceleration (impact). This resulted in false positives during quickly standing up. The reason for this is that the quickly standing up has also a high acceleration and can be confused with a fall. However, the pattern during standing up is a reverse compared to the fall pattern: first the increase is detected followed by the decrease. Using this information the minimum and the maximum acceleration within 1.5 second window were measured. If the difference between the maximum and the minimum exceeded the threshold and the maximum (impact) appeared after the minimum (fall), we declared that a fall had occurred. The threshold was chosen empirically for each of the accelerometers individually. Eventually the waist accelerometer had the threshold of $0.8 g$ and chest accelerometer had $1 \mathrm{~g}$. The reason for this is the placement of the accelerometers on the body and the impact with the ground. This method works perfectly on normal fast falls, and the false alarms rate during normal activities is reduced to minimum.

The problem with this algorithm appears if there is high acceleration (decrease followed by increase) but the event is not fall (e.g. quickly sitting on the chair). To solve this issue we used a second algorithm, which takes into account the recognized posture after a potential fall event. The improved algorithm developed for detecting fall events used the postures recognized from the posture recognition module as input. It analyzed the recognized postures and decided if a fall was detected. Two rules were implemented. The fall had occurred if:

- Acceleration has exceeded the threshold as described in the first algorithm AND the person is lying for more than 10 seconds; OR

- The person sits on the ground more than 10 seconds.

The assumption of the second rule, which is already implicit in the scenario, was that the elderly usually do not sit on the ground.

The decision about the posture of the person in 10 seconds interval was done by choosing the major predicted posture in this interval. In other words, the posture with highest number of data samples was chosen.

\section{EXPERIMENTAL RESULTS}

We compared the performances of the posture recognition and fall detection methods on a test scenario. It was recorded by 11 healthy volunteers ( 7 male and 4 female), 5 times by each person. We made a comparison to a location system. This was possible because each person was wearing four accelerometers and four location tags during the recordings.

\section{A. Test Scenario}

The test scenario is around 15 minutes long and includes all the target body postures. It was designed specifically to investigate events that may be difficult to recognize as falls or non-falls. The events are listed in Table 1 . They were recorded in single recordings interspersed with short periods of walking.

TABLE 1: EVENTS SEQUENCE IN THE TEST SCENARIO.

\begin{tabular}{|c|c|}
\hline No. & Description \\
\hline 1 & Sitting down normally on the chair \\
\hline 2 & Tripping, falling fast on the ground \\
\hline 3 & Lying down normally on the bed \\
\hline 4 & $\begin{array}{l}\text { Falling slowly (trying to hold onto furniture), lying } \\
\text { on the ground }\end{array}$ \\
\hline 5 & Sitting down quickly on the chair \\
\hline 6 & $\begin{array}{l}\text { Falling from chair slowly when trying to stand up } \\
\text { (trying to hold onto furniture), landing sitting of } \\
\text { the ground }\end{array}$ \\
\hline 7 & Sitting down quickly on the chair \\
\hline 8 & $\begin{array}{l}\text { Falling from chair quickly when trying to stand up, } \\
\text { landing sitting of the ground }\end{array}$ \\
\hline 9 & $\begin{array}{l}\text { Searching for something on the ground - on all } \\
\text { fours and lying }\end{array}$ \\
\hline
\end{tabular}

As shown in the section on related work, accelerometers can accurately detect typical falls, so we included only one such fall (event number 2) to demonstrate that the system can recognize it accurately. We included two atypical falls (4, 6 and 8 ) to test the use of posture information, namely that a person is not expected to sit on the ground (as opposed to the chair). Furthermore, we included two events (5 and 7) that involve high acceleration and could thus be misclassified as falls by accelerometers. We also included an event (9) that involves voluntary lying on the ground, which could mislead the methods that use information other than acceleration. The last two events (1 and 3) are perfectly normal and were included to verify that all the methods work correctly and do not recognize them as falls.

\section{B. Results}

The results are shown for each of the modules in separate and different ways. For body posture recognition the results are presented as the accuracy of the classifier for each of the class values (postures). The confusion matrix for the waist accelerometer is also shown. The results for fall detection are shown in terms of events from the test scenario.

\section{1) Body Posture Recognition}

For body posture recognition module, leave one person out technique was used for evaluating the results. That means the model was trained on 10 people and tested on the remaining person. This procedure was done 11 times, once for each person. At the end the average accuracy was calculated for each posture separately and the overall accuracy was calculated for all the postures together. This evaluation approach is more reliable than the ones that use separate testing scenarios for each activity (only standing, only lying) or that use the same person for training and testing. Using the same person would give overly optimistic results if the intended use of the model is to classify the postures of previously unseen people. As we mentioned before every data sample is classified. That means for one event of lying on the 
bed for 10 seconds there are 600 samples that should be classified as lying. Therefore the possibility for errors in a few samples at the beginning and at the end of each event is high.

We compared the accuracy of the accelerometer-based posture recognition to a location system consisted of four wearable tags. We did not train this system on our data. Instead, we used a classification model included in the system and just run it on the newly recorded data (11 people). The model was previously built on a similar scenario.

The results of this posture recognition module are shown in Table 3. Because there is a lot of related work that presents accuracies using different numbers and placements of accelerometers, we decided to include nine different sensor placements in our table. It should be noted that accelerometers placed only on the thigh or ankle did not achieve reasonable results, so they are omitted from the final table. The results showed that the chest and waist accelerometers are best suited to posture recognition. Since the chest accelerometer is more difficult to wear, we wished to test whether it can be successfully replaced by the waist accelerometer. From the results that we got, we concluded that by replacing the chest with waist accelerometer the system loses a few percents in the overall accuracy.

If we analyze the confusion matrix for the waist accelerometer shown in Table 2, we can make several conclusions. For system with only one accelerometer (chest or waist):

- It is difficult to distinguish sitting and standing, or sitting and sitting on the ground. This is because the orientation of the sensor is similar during these static postures.

- On all fours is usually confused with lying on the stomach, because the sensor orientation is the same (parallel to the ground and facing the ground).

- Transitional activities are difficult to distinguish and are usually mutually misclassified or confused with dynamic standing - walking. The small errors in other postures are because of the mistakes in manual data labeling.
TABLE 2: CONFUSION MATRIX FOR THE WAIST ACCELEROMETER. LYING (LY), SITTING (SIT), STANDING (STA), ON ALl FOURS (ON4), SITTING ON THE GROUND (SITG), GOING DOWN (GD), STANDING UP (SU)

\begin{tabular}{|c|c|c|c|c|c|c|c|}
\hline Waist & \multicolumn{7}{|c|}{ Classified as } \\
\hline Posture & Ly & Sit & Sta & On4 & SitG & GD & SU \\
\hline Ly & $\mathbf{9 4 \%}$ & $\mathbf{0 \%}$ & $\mathbf{0 \%}$ & $\mathbf{1 \%}$ & $\mathbf{0 \%}$ & $\mathbf{2 \%}$ & $\mathbf{3 \%}$ \\
\hline Sit & $\mathbf{0 \%}$ & $\mathbf{6 6 \%}$ & $\mathbf{1 4 \%}$ & $\mathbf{0 \%}$ & $\mathbf{1 9 \%}$ & $\mathbf{0 \%}$ & $\mathbf{1 \%}$ \\
\hline Sta & $\mathbf{0 \%}$ & $\mathbf{1 1 \%}$ & $\mathbf{8 4 \%}$ & $\mathbf{0 \%}$ & $\mathbf{3 \%}$ & $\mathbf{1 \%}$ & $\mathbf{1 \%}$ \\
\hline On4 & $\mathbf{5 0 \%}$ & $\mathbf{0 \%}$ & $\mathbf{0 \%}$ & $\mathbf{4 4 \%}$ & $\mathbf{0 \%}$ & $\mathbf{0 \%}$ & $\mathbf{6 \%}$ \\
\hline SitG & $\mathbf{0 \%}$ & $\mathbf{3 8 \%}$ & $\mathbf{9 \%}$ & $\mathbf{0 \%}$ & $\mathbf{5 3 \%}$ & $\mathbf{0 \%}$ & $\mathbf{0 \%}$ \\
\hline GD & $\mathbf{3 \%}$ & $\mathbf{1 \%}$ & $\mathbf{2 6 \%}$ & $\mathbf{5 \%}$ & $\mathbf{1 \%}$ & $\mathbf{3 9 \%}$ & $\mathbf{2 6 \%}$ \\
\hline SU & $\mathbf{5 \%}$ & $\mathbf{1 \%}$ & $\mathbf{9 \%}$ & $\mathbf{5 \%}$ & $\mathbf{1 \%}$ & $\mathbf{1 5 \%}$ & $\mathbf{6 4 \%}$ \\
\hline
\end{tabular}

If we return to Table 3 , another thing that is obvious is a big improvement in accuracy when the system is using two accelerometers. When the chest or waist accelerometer is combined with the ankle accelerometer, there is a noticeable improvement in sitting and sitting on the ground. When it is combined with the thigh accelerometer, there is an improvement in distinguishing between sitting and standing, and also lying and on all fours. The orientation of the thigh sensor in both cases is parallel vs. perpendicular to the ground.

With introducing the third accelerometer there is an improvement in each of the postures, and with 4 accelerometers the system is almost perfect. It should be emphasized that $100 \%$ accuracy is almost impossible because of the previously discussed issue with the labeling.

If we compare accelerometers to body posture recognition using location sensors, we see that results using only 2 accelerometers outperform 4 location tags in almost any posture. Three accelerometers offer 7-9 percentage points better accuracy.

TABlE 3: COMPARISON OF POSTURE RECOGNITION ACCURACY USING DIFFERENT NUMBER OF ACCELEROMETERS (1, 2, 3 OR 4) PLACED on the Chest (C), Waist (W), Ankle Right (AR) and Thigh Right (TR) and using 4 location tags

\begin{tabular}{|c|c|c|c|c|c|c|c|c|c|c|}
\hline Posture & $\mathrm{C}$ & $\begin{array}{c}\mathrm{C}+ \\
\mathrm{AR}\end{array}$ & $\begin{array}{c}\mathrm{C}+ \\
\mathrm{TR}\end{array}$ & $\begin{array}{c}\mathrm{C}+\mathrm{AR} \\
+\mathrm{TR}\end{array}$ & $\mathrm{W}$ & $\mathrm{W}+\mathrm{AR}$ & $\mathrm{W}+\mathrm{TR}$ & $\begin{array}{c}\mathrm{W}+\mathrm{AR} \\
+\mathrm{TR}\end{array}$ & $\begin{array}{c}\mathrm{C}+\mathrm{W}+ \\
\mathrm{AR}+\mathrm{TR}\end{array}$ & $\begin{array}{c}4 \mathrm{Location} \\
\text { tags }\end{array}$ \\
\hline Lying & $\mathbf{9 8 \%}$ & $\mathbf{9 9 \%}$ & $\mathbf{9 9 \%}$ & $\mathbf{9 9 \%}$ & $\mathbf{9 4 \%}$ & $\mathbf{9 7 \%}$ & $\mathbf{9 6 \%}$ & $\mathbf{9 7 \%}$ & $\mathbf{9 9 \%}$ & $\mathbf{9 3 \%}$ \\
\hline Sitting & $\mathbf{4 9 \%}$ & $\mathbf{7 7 \%}$ & $\mathbf{8 9 \%}$ & $\mathbf{9 6 \%}$ & $\mathbf{6 6 \%}$ & $\mathbf{8 2 \%}$ & $\mathbf{9 7 \%}$ & $\mathbf{9 8 \%}$ & $\mathbf{9 9 \%}$ & $\mathbf{8 9 \%}$ \\
\hline Standing & $\mathbf{8 1 \%}$ & $\mathbf{9 3 \%}$ & $\mathbf{9 9 \%}$ & $\mathbf{9 9 \%}$ & $\mathbf{8 4 \%}$ & $\mathbf{8 3 \%}$ & $\mathbf{9 8 \%}$ & $\mathbf{9 9 \%}$ & $\mathbf{9 9 \%}$ & $\mathbf{9 4 \%}$ \\
\hline On all fours & $\mathbf{4 9 \%}$ & $\mathbf{5 3 \%}$ & $\mathbf{8 8 \%}$ & $\mathbf{9 3 \%}$ & $\mathbf{4 4 \%}$ & $\mathbf{4 8 \%}$ & $\mathbf{8 4 \%}$ & $\mathbf{9 2 \%}$ & $\mathbf{9 7 \%}$ & $\mathbf{2 2 \%}$ \\
\hline Sit on ground & $\mathbf{5 3 \%}$ & $\mathbf{9 5 \%}$ & $\mathbf{8 8 \%}$ & $\mathbf{9 3 \%}$ & $\mathbf{5 3 \%}$ & $\mathbf{8 6 \%}$ & $\mathbf{8 6 \%}$ & $\mathbf{9 2 \%}$ & $\mathbf{9 6 \%}$ & $\mathbf{4 4 \%}$ \\
\hline Going down & $\mathbf{4 5 \%}$ & $\mathbf{5 4 \%}$ & $\mathbf{5 4 \%}$ & $\mathbf{6 6 \%}$ & $\mathbf{3 9 \%}$ & $\mathbf{4 7 \%}$ & $\mathbf{4 9 \%}$ & $\mathbf{5 5 \%}$ & $\mathbf{7 5 \%}$ & $\mathbf{4 2 \%}$ \\
\hline Standing up & $\mathbf{6 7 \%}$ & $\mathbf{7 3 \%}$ & $\mathbf{7 9 \%}$ & $\mathbf{8 2 \%}$ & $\mathbf{6 4 \%}$ & $\mathbf{7 0 \%}$ & $\mathbf{7 8 \%}$ & $\mathbf{7 8 \%}$ & $\mathbf{8 2 \%}$ & $\mathbf{7 4 \%}$ \\
\hline Overall & $\mathbf{7 5 \%}$ & $\mathbf{9 1 \%}$ & $\mathbf{9 3 \%}$ & $\mathbf{9 8 \%}$ & $\mathbf{7 7 \%}$ & $\mathbf{8 9 \%}$ & $\mathbf{9 3 \%}$ & $\mathbf{9 6 \%}$ & $\mathbf{9 9 \%}$ & $\mathbf{8 9 \%}$ \\
\hline
\end{tabular}




\section{2) Fall Detection}

We compared the performance of fall detection using posture recognition with all the sensor placements. Table 4 shows the accuracies of the recognition of the four falls and the three potentially misleading events described in the previous subsection. Both events consisting of sitting down quickly are merged into one line. The average accuracy was averaged over the seven events. The accuracies of the two normal events ( 1 and 3 in Table 1 ) are not included explicitly; instead, the total number of other false alarms (activities incorrectly recognized as falls) during these two events and during the walking between the events is given.

The first event in Table 4, tripping, is a typical fall that was recognized fairly accurately by all algorithms. This is the event that is mostly detected by the methods described in related work.

Falling slowly was difficult to recognize using only accelerometers, because the falling was too slow and lying itself is not alarming. Such fall can be recognized if one knows where the bed is and that a person is not supposed to lie outside the bed. Such information can be extracted using location sensors or simple pressure sensors installed on the bed (and the chair).

Falling from chair slowly was difficult to recognize using only the acceleration threshold. The reason is the low acceleration during this event. During the second (quickly) falling from chair the acceleration is bigger, which resulted in better accuracy. The improved method, which uses the posture, was able to detect these events with higher accuracy, because the posture of the person was recognized as sitting on the ground for more than 10 seconds. Some problems emerged when using only 1 accelerometer because the accuracy of the recognition of sitting on the ground posture is little more than $50 \%$. Location sensors also performed well because they could recognize sitting, and they had the information about the location, which was not the chair.

The two sittings on the chair quickly were easy to recognize for the location-based system because it knew that they were taking place on the chair. The algorithm using acceleration threshold only had problems with this event, because the acceleration was high and the pattern was similar to fall. The improved algorithm recognized sitting quickly quite accurately, because posture recognition (sitting) correctly indicated that no fall had occurred.

In the case of searching on the ground, the knowledge of the location was a disadvantage, because the posture was similar to lying and the location was not the bed. However, the acceleration-based algorithms were $100 \%$ accurate.

The false alarms appeared only in the improved algorithm with only one accelerometer. The problem occurred while the person was sitting on the chair and the posture recognition module incorrectly recognized sitting on the ground.

Looking at the average accuracies and other false alarms, one can make the following conclusions:

- There is a big improvement using 2 instead of 1 accelerometer. But there was a slight difference in accuracy of using 3 or 4 instead of 2 . One can make a decision by comparing the price of the system and the comfort of wearing on one side, and the accuracy on the other side.

- There is no big difference between the chest and the waist accelerometer. Because the chest tag is more difficult to wear, the waist tag may be preferable for fall detection.

TABLE 4: FALL DETECTION ACCURACY OF THE ACCELEROMETER-BASED METHODS:

THRESHOLD AND THRESHOLD + BODY POSTURE RECOGNITION FOR FALL AND NON-FALL EVENTS (EVENT NUMBERS FROM TABLE 1 ARE IN PARENTHESES). ACCElEROMETER PlACEMENTS - Chest (C), WAist (W), ANKLE Right (AR) AND THIGH Right (TR).

\begin{tabular}{|c|c|c|c|c|c|c|c|c|c|c|c|c|}
\hline \multirow[b]{2}{*}{ Events } & \multicolumn{2}{|c|}{ Threshold } & \multicolumn{9}{|c|}{ Threshold + Body Posture Recognition } & \multirow[b]{2}{*}{$\begin{array}{c}4 \\
\text { Location } \\
\text { Sensors }\end{array}$} \\
\hline & $\mathrm{C}$ & $\mathrm{W}$ & $\mathrm{C}$ & $\mathrm{W}$ & $\begin{array}{l}\mathrm{C}+ \\
\mathrm{AR}\end{array}$ & $\begin{array}{l}\mathrm{C}+ \\
\mathrm{TR}\end{array}$ & $\begin{array}{l}\text { W+ } \\
\text { AR }\end{array}$ & $\begin{array}{l}\mathrm{W}+ \\
\mathrm{TR}\end{array}$ & $\begin{array}{c}\mathrm{C}+ \\
\mathrm{AR}+ \\
\mathrm{TR}\end{array}$ & $\begin{array}{c}\mathrm{W}+ \\
\mathrm{AR}+ \\
\mathrm{TR} \\
\end{array}$ & $\begin{array}{l}\mathrm{C}+\mathrm{W} \\
+\mathrm{AR} \\
+\mathrm{TR}\end{array}$ & \\
\hline \multicolumn{13}{|l|}{ Falls } \\
\hline Tripping & $100 \%$ & $100 \%$ & $100 \%$ & $100 \%$ & $100 \%$ & $100 \%$ & $100 \%$ & $100 \%$ & $100 \%$ & $100 \%$ & $100 \%$ & $100 \%$ \\
\hline Falling slowly & $11 \%$ & $8 \%$ & $11 \%$ & $8 \%$ & $11 \%$ & $11 \%$ & $8 \%$ & $8 \%$ & $11 \%$ & $8 \%$ & $11 \%$ & $98 \%$ \\
\hline $\begin{array}{l}\text { Falling from } \\
\text { chair slowly }\end{array}$ & $17 \%$ & $11 \%$ & $63 \%$ & $66 \%$ & $96 \%$ & $92 \%$ & $93 \%$ & $90 \%$ & $100 \%$ & $100 \%$ & $100 \%$ & $92 \%$ \\
\hline $\begin{array}{l}\text { Falling from } \\
\text { chair quickly }\end{array}$ & $49 \%$ & $38 \%$ & $72 \%$ & $71 \%$ & $95 \%$ & $93 \%$ & $93 \%$ & $92 \%$ & $100 \%$ & $100 \%$ & $100 \%$ & $96 \%$ \\
\hline Non-falls & \multirow[b]{2}{*}{$36 \%$} & \multirow[b]{2}{*}{$33 \%$} & \multirow[b]{2}{*}{$82 \%$} & \multirow[b]{2}{*}{$82 \%$} & \multirow[b]{2}{*}{$90 \%$} & \multirow[b]{2}{*}{$96 \%$} & \multirow[b]{2}{*}{$92 \%$} & & \multirow[b]{2}{*}{$100 \%$} & & \multirow[b]{2}{*}{$100 \%$} & \multirow[b]{2}{*}{$100 \%$} \\
\hline sit quickly & & & & & & & & $100 \%$ & & $100 \%$ & & \\
\hline on all fours & $100 \%$ & $100 \%$ & $100 \%$ & $100 \%$ & $100 \%$ & $100 \%$ & $100 \%$ & $100 \%$ & $100 \%$ & $100 \%$ & $100 \%$ & $80 \%$ \\
\hline Average & $52 \%$ & $48 \%$ & $71 \%$ & $71 \%$ & $82 \%$ & $82 \%$ & $81 \%$ & $82 \%$ & $85 \%$ & $85 \%$ & $85 \%$ & $94 \%$ \\
\hline $\begin{array}{l}\text { Other false } \\
\text { alarms }\end{array}$ & 0 & 0 & 5 & 5 & $\mathbf{0}$ & $\mathbf{0}$ & 0 & 0 & 0 & 0 & 0 & 2 \\
\hline
\end{tabular}




\section{CONCLUSION}

We investigated the impact of accelerometer number and placement on the accuracy of posture recognition. Our goal was to recognize seven postures. We compared the results to a system using four ultra-wideband location tags. With one accelerometer placed on the chest or waist, the classification model was able to distinguish between two groups of postures (\{lying and on all fours $\}$ and \{standing, sitting and sitting on the ground $\}$ ). This is because of the difference in sensor angle orientation. The problems emerged while distinguishing postures within each group. When including one more accelerometer in the system, the overall accuracy improved up to $90 \%$ with almost any sensor combination. This is already better than $89 \%$ achieved with the location system with 4 tags. Three accelerometers improved the accuracy for 3-5 percentage points (depending on the sensor combination) and by including the fourth accelerometer the accuracy increased to $99 \%$. Our experiments proved that accelerometers are superior to location sensors in posture recognition task. Furthermore the location sensor system is more expensive and also requires installation in the apartment. The chest and waist are the most common places to put an accelerometer and a comparison of the two revealed that they perform very similarly, with the chest having a small advantage.

The posture recognition methods were used to improve fall detection by recognizing whether the posture could be the result of a fall. We again investigated the impact of accelerometer number and placement. Using one accelerometer on the chest or waist improved the thresholdbased method for 20 percentage points overall. Significant improvements appeared in the falls ending with sitting on the ground and non-falls ending with sitting. Two accelerometers (each of the analyzed sensor combinations) improved the results for $10-11$ percentage points mostly because of the correctly recognizing the posture sitting on the ground. Including the third accelerometer (in both cases) the system achieved overall accuracy of $85 \%$. Using all four accelerometers didn't improve the overall accuracy.

In fall detection the waist accelerometer proved to have almost same performances as the chest accelerometer. So, one could choose the waist because wearing an accelerometer there is more comfortable.

In fall detection the location sensors proved to be better, but only because they could use the knowledge about the location of the bed and chair. It was very hard for accelerometers to distinguish slowly falling down and lying on the bed using only acceleration signals. The accelerometers fall detection performance can be improved by the information about the location where a potential fall is taking place. In future we plan to include this information in the system, thus the events that end up with lying outside the bed or sitting outside the chair would probably be correctly recognized as falls.

The second issue for further work is developing more sophisticated methods for recognizing the transitional activities/postures (standing up, going down). This may make it possible to distinguish between standing and sitting with only one accelerometer.

\section{ACKNOWLEDGMENT}

The research leading to these results has received funding from the European Community's Framework Programme FP7/2007-2013 under grant agreement nº 214986.

\section{REFERENCES}

[1] Nishkam Ravi, Nikhil Dandekar, Preetham Mysore and Michael L. Littman: "Activity recognition from accelerometer data", IAAI'05 Proceedings of the 17th conference on Innovative applications of artificial intelligence - Volume 3 .

[2] Zhenyu He, Lianwen Jin: "Activity recognition from acceleration data based on discrete consine transform and SVM", Proceedings of the 2009 IEEE International Conference on Systems, Man, and Cybernetics San Antonio, TX, USA - October 2009.

[3] V. Mirchevska, M. Luštrek, and M. Gams, "Combining machine learning and expert knowledge for classifying human posture", Proc. of International Electrotechnical and Computer Science Conference 2009, pp. 183-186, 2009.

[4] Nguyen, T.-T., Cho, M.-C., and Lee, T.-S. "Automatic fall detection using wearable biomedical signal measurement terminal". In Proceedings of 31 st Annual International Conference of the IEEE EMBS, 5203-5206, 2009.

[5] Li, Q., Stankovic, J. A., Hanson, M. A., Barth, A. T., Lach, J., and Zhou, G. "Accurate, fast fall detection using gyroscopes and accelerometer-derived posture information. In 2009 Proceedings of Sixth International Workshop.

[6] Shadi Khawandi, Bassam Daya \& Pierre Chauvet, "Automated monitoring system for fall detection in the elderly", International Journal of Image Processing (IJIP), Volume (4): Issue (5), 2010.

[7] M. Kangas, A. Konttila, I. Winblad, and T. Jamsa, "Determination of simple thresholds for accelerometry-based parameters for fall detection," Proc. 29th Annual Int'l Conf. Engineering in Medicine and Biology Society (EMBS 2007), IEEE CS Press, 2007, pp. 1367-1370.

[8] i2010 - A European Information Society for growth and employment. http://ec.europa.eu/information_society/eeurope/i2010/index_en.htm.

[9] Luštrek, M., and Kaluža, B. "Fall detection and activity recognition with machine learning". Informatica 33(2): 197-204, 2009.

[10] Kaluža, B., Mirchevska, V., Dovgan, E., Luštrek, M., and Gams, M. "An agent-based approach to care in independent living". In Proceedings of AmI 2010, 177-186, 2010.

[11] Confidence project: http://www.confidence-eu.org/.

[12] Jantaraprim, P., Phukpattaranont, P., Limsakul, C., and Wongkittisuksa, B. "Evaluation of fall detection for the elderly on a variety of subject groups". In Proceedings of i-CREATe 2009.

[13] Chin-Feng Lai, Yueh-Min Huang, Han-Chieh Chao, and Jong Hyuk Park, "Adaptive Body Posture Analysis Using Collaborative MultiSensors for Elderly Falling Detection", IEEE Intelligent Systems (SCI), IEEE CS, Vol. 25, Iss. 2, pp. 20-30,2010.

[14] Zhang, T., Wang, J., Liu, P., and Hou, J. 2006. Fall Detection by Wearable Sensor and One-Class SVM Algorithm. In Lecture Notes in Control and Information Science 345, 858-863.

[15] Shan, S., and Yuan, T. 2010. A Wearable Pre-Impact Fall Detector Using Feature Selection and Support Vector Machine. In Proceedings of ICSP'10, 1686-1689. 